\title{
Steady State Modification Method Based On Backpropagation Neural Network For Non- Intrusive Load Monitoring (NILM)
}

\author{
Sigit Tri Atmaja ${ }^{1, *}$, Abdul Halim ${ }^{1}$ \\ ${ }^{1}$ Department of Electrical Engineering, Faculty of Engineering, Universitas Indonesia, Depok, \\ Indonesia
}

\begin{abstract}
Household electric power sector is highlighted as one of significant contributors to national energy consumption. To reduce electric energy usage in this sector, a technique called Non-Intrusive Load Monitoring (NILM) has been developed recently. NILM is a load disaggregating and monitoring tool that can be used to identify the daily usage behavior of individual electric appliance. Different to conventional method, NILM promises the reduction of sensor deployment significantly. NILM commonly uses either transient or steady state signal. Based on load/appliance signal condition, many NILM's research results have been published. In this paper, steady state modification method of backpropagation neural network (NN) is applied for developing NILM. We use steady state signal to disaggregate the sum of load power signal. In the proposed method, $\mathrm{NN}$ is explored for feature extraction of electric power consumption of individual appliance. The presented method is powerful for load power signal which has almost same value. To verify the effectiveness of proposed method, data provided by tracebase.org has been used. The presented method can be applied for local data. It is obvious from simulation results that the proposed method could improve the recognition rate of appliances until $100 \%$.
\end{abstract}

\section{Introduction}

Mankind is facing energy problem seriously. Energy consumption is growing rapidly; however, the depletion of fossil energy resources is unstoppable. Nowadays, the average growth of electrical energy consumption is about $2.3 \%-2.5 \%$, and by the end of 2030 it is estimated that the increasing will be twice or around 16,000 TWh per year [1]. The growth of energy consumption will give a negative impact on the environment such as increasing of Carbon dioxide (CO2), Nitrogen oxide (NOx), and Sulfur dioxide (SOx) emission. Many researchers are developing technologies in order to address this problem like renewable energy technologies, environmentally friendly material technologies, energy efficient appliances etc. [2].

\footnotetext{
* Corresponding author: sigit.tri@ui.ac.id
} 
Excessive consumption of energy takes place in various sectors. One of them is household sector. Electric power use in this sector has been underlined as significant contributor to national energy consumption. Efforts to reduce energy consumed in residential have been exercised by introducing a tool to monitor electric power consumption and give this information back to consumers [3,4]. Generally, conventional load monitoring system uses a lot of sensors mounted on each appliance. Thus, it is very high cost both for installation and maintenance and not practice for household application [5].

Dissimilar to conventional one, nowadays, development of energy monitoring system has been focused to develop a technique that be able to minimize sensor number. And NonIntrusive load monitoring (NILM) has become iconic research result. NILM is a method that can disaggregate electrical load changes and then identify the daily usage behavior of individual electric appliance $[5,6]$. The advantage and disadvantage of NILM method can be summarized as follows [7]:

- Advantages: Low cost and ease of installation and maintenance, No needs large space for mounting, and Able to cover large number of appliances to be monitored,

- Disadvantages: Continuously variable appliance cannot be detected, Electrically identical appliances cannot be distinguished, Greater potential for undetected error, and difficult to recognize unusual appliances.

Various NILM techniques have been proposed. Some mathematical models have been explored such as Hidden Markov Model (HMM), Fuzzy Model, Graph Signal Processing, Linear Programming Model etc. One of the powerful models is Neural network (NN). Many research publications have discussed the advantages of artificial neural networks to improve the performance of NILM system. Some improved neural network methods have been presented to identify the load signal on transient [8] and steady state conditions [9]. In our research, an improved type of backpropagation $\mathrm{NN}$ is exercised for developing more efficient and effective NILM method. NN in the proposed method is utilized for feature extraction of individual electric power consumption.

In our research, an input layer of $\mathrm{NN}$ is added to overcome the difficulty when there is a steady state signal which has almost same value. That additional input layer is set for maximum power value of appliance during switch on. By using simulation, the proposed method has proved that recognition rate could be improved even when the active power of each appliances has almost same value.

\section{Methodology}

\subsection{Problem Definition}

The main idea of NILM system is to calculate the power consumption of individual appliances in a house based on the analysis of the aggregated data measured from a single meter. The total aggregated measurement of the household power consumption of the individual appliance from the main meter can be estimated according to equation [7]

$$
p_{t}=\sum_{i=1}^{n} p_{i}(t)+e_{t}
$$

where $p_{t}$ is aggregated household power consumption, $p_{i}(t)$ is individual appliance power consumption at a given period of time and is measurement errors and line loss. Specific characteristic of power signal appears in each electrical appliance during the normal working cycle. This characteristic contains information about values of electrical parameters during steady-state or transient states. The aggregate power signal from the main meter is shown in Figure 1. This graphic represents steady state condition. This curve is derived from database of tracebase [11]. 


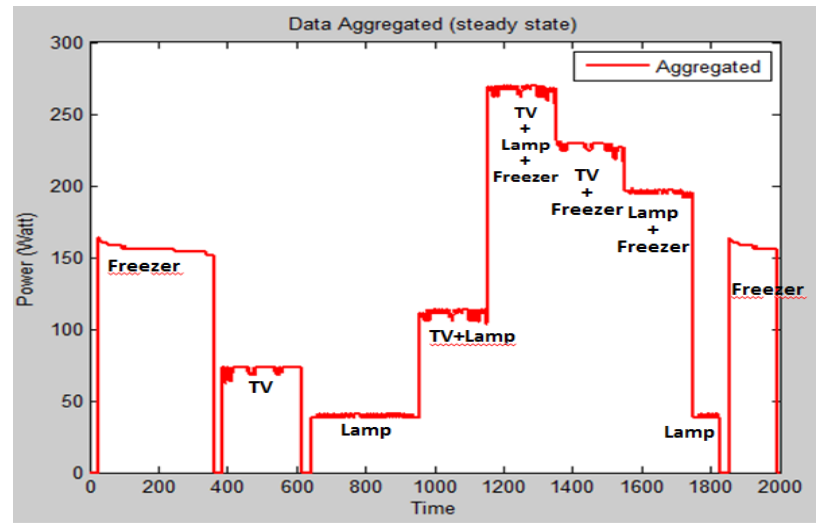

Fig. 1. Aggregated Power Signal

As shown in Figure 1, power signal level at each period of time has significant different. In this case, NILM system using neural network can work well. However, when power signal level has almost same value at different period as depicted in Figure 2, the problem of $\mathrm{NN}$ application has occurred. In this paper, a modified method for overcoming this challenge is provided.

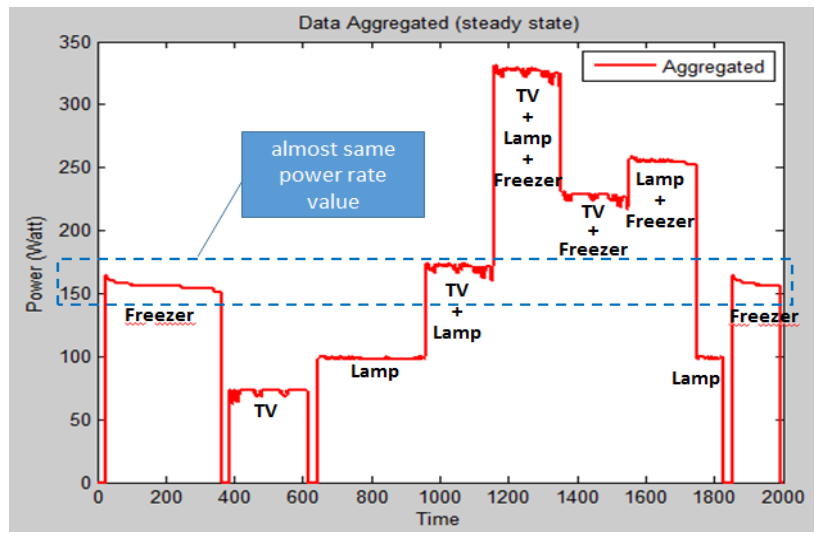

Fig. 2. Aggregated Signal With Almost Same Power Level

\subsection{Steady state method Modification}

Steady state method can be classified as conventional and modification type. Each type is described in this section.

\subsubsection{Conventional Type}

In this section, we present two types of $\mathrm{NN}$ for disaggregating the load. Firstly, the conventional type is urged. This method uses one neuron on input layer of neural network architecture. Active power is set as the input layer. The architecture of the backpropagation neural network can be seen in detail in Figure 3 below. In general, the backpropagation neural network structure consists of three layers: the input layer, the hidden layer and the output layer including the neuron[10]. 


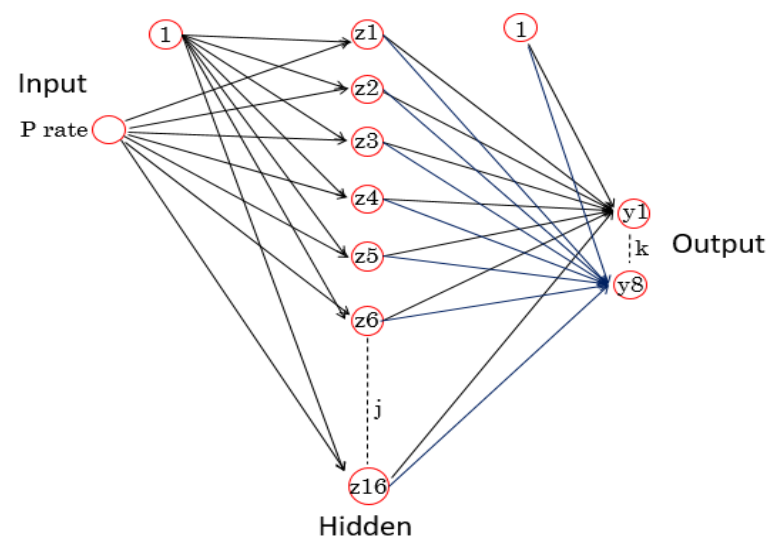

Fig. 3. Architecture NN with steady state method

The hidden layer has 16 neuron and the output layer has 8 neuron. This output layer gives information about the status of each appliance. Output layer contain 1 if the appliance switch on and vice versa. Detail of each output layer can be seen in the Table 1 below.

Table 1. Output Layer

\begin{tabular}{|c|c|c|c|c|c|c|c|c|}
\hline \multirow{2}{*}{$\begin{array}{c}\text { Appliances } \\
\text { ON }\end{array}$} & \multicolumn{7}{|c|}{ Neuron of Neural Network Output Layer } \\
\cline { 2 - 10 } & $\boldsymbol{y} \mathbf{1}$ & $\boldsymbol{y} \mathbf{2}$ & $\boldsymbol{y 3}$ & $\boldsymbol{y} \boldsymbol{4}$ & $\boldsymbol{y} \mathbf{5}$ & $\boldsymbol{y} \boldsymbol{6}$ & $\boldsymbol{y} \boldsymbol{7}$ & $\boldsymbol{y} \boldsymbol{8}$ \\
\hline Freezer & 1 & 0 & 0 & 0 & 0 & 0 & 0 & 0 \\
\hline TV & 0 & 1 & 0 & 0 & 0 & 0 & 0 & 0 \\
\hline Lamp & 0 & 0 & 1 & 0 & 0 & 0 & 0 & 0 \\
\hline Freezer+TV & 0 & 0 & 0 & 1 & 0 & 0 & 0 & 0 \\
\hline Freezer+ Lamp & 0 & 0 & 0 & 0 & 1 & 0 & 0 & 0 \\
\hline TV+Lamp & 0 & 0 & 0 & 0 & 0 & 1 & 0 & 0 \\
\hline Freezer+TV+Lamp & 0 & 0 & 0 & 0 & 0 & 0 & 1 & 0 \\
\hline OFF & 0 & 0 & 0 & 0 & 0 & 0 & 0 & 1 \\
\hline
\end{tabular}

The simulation of backpropagation neural network is designed using MATLAB. The specification of this neural network are as follows : maximum epoch 2000, target MSE value $<1 \mathrm{e}-06$, the amount of learning data per probability of appliance when turned on is 1000 , and the value for (alpha) is set to 0.3. The result of the learning is the value of neural network weights. Iteration learning will stop when it has achieved the target of mean square error (MSE) or maximum epoch value. The MSE is the performance index function commonly used to learn feed forward neural networks. The error is occurred between the output neural network and the target output. MSE can be computed by

$$
M S E=\frac{1}{n} \sum_{i=1}^{n}\left(\hat{Y}_{i}-Y_{i}\right)^{2}
$$

where variable $n$ is the number of learning samples, variable $\hat{Y}_{i}$ is the target output, and variable $Y_{i}$ is neural network output. After the value of the weight has get from learning neural network, the testing of NN is carried out. The testing of NN results Recognition Rate (RR) value.

\subsubsection{Modification Type}

The difference between the previous type is an additional one input layer set on an input layer. That additional input layer is the maximum power of appliance. Hence, this type uses 
two neuron on input layers. Detailed this architecture of the backpropagation neural network can be seen in Figure 4 below.

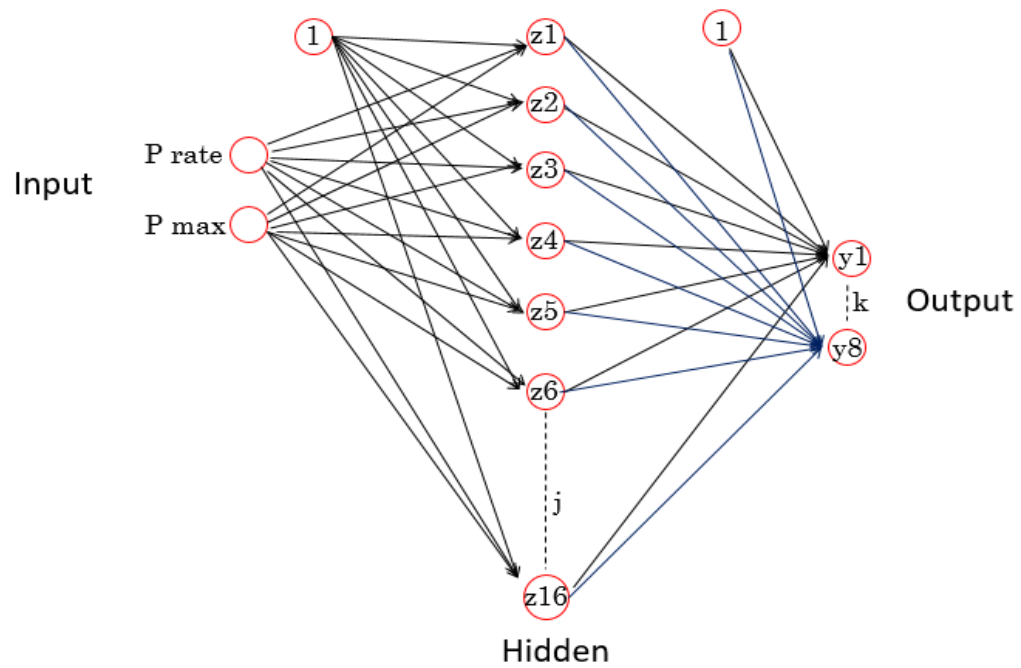

Fig. 4. Architecture NN with steady state modification method

\section{Simulation and Analysis}

Before starting learning of backpropagation $\mathrm{NN}$, data for input layer should be normalized firstly. In this study, data for learning normalized between minimum 0 and maximum 1 using min max formula.

\subsection{Simulation using steady state method}

In this simulation, we use aggregated data with significant differences of power rate value. The results of learning backpropagation $\mathrm{NN}$ with steady state method on this data using same specification parameter of learning. The MSE value of learning backpropagation $\mathrm{NN}$ is 0.0015 and the epoch value is 2000 . For testing the results of RR is $100 \%$. Other experiment has done by using data which has the almost same power rate value. The MSE value of learning backpropagation $\mathrm{NN}$ is 0.051 and the epoch value is 2000. Moreover, RR value of this testing is $81.9 \%$.

\subsection{Steady State Modification Method}

In this study, modification type of NN is tried for performance verification. Simulating is performed using aggregated data with almost the same power rate value. We use the same NN learning specifications. The results the MSE value of learning NN is 4.0426e-06 and the epoch value is 2000 . RR for testing is $100 \%$.

Table 2. Result Of Simulation

\begin{tabular}{|c|c|c|c|c|c|c|}
\hline & \multicolumn{3}{|c|}{ Data : significant difference power rate } & \multicolumn{3}{|c|}{ Data : almost the same power rate } \\
\hline & $\begin{array}{c}\text { MSE } \\
\text { Learning }\end{array}$ & Epoch & $R R(\%)$ & $\begin{array}{c}\text { MSE } \\
\text { Learning }\end{array}$ & Epoch & $R R(\%)$ \\
\hline Steady State & 0.0015 & 2000 & 100 & 0.051 & 2000 & 81.9 \\
\hline $\begin{array}{l}\text { Modify } \\
\text { Steady State }\end{array}$ & - & - & - & $4.0426 \mathrm{e}-06$ & 2000 & 100 \\
\hline
\end{tabular}


The above simulation result is summarized in Table 2. It can be stated that the proposed modification method proves the significant improvement compared with conventional one. In this paper, the data used is provided by foreign institution which is tracebase. However, the above method can be applied for domestic data. Now we are preparing for providing power signal of local appliance.

\section{Conclusion}

Based on the result of simulation, it shows that steady state modification method has significant performance for identifying or disaggregating each appliances. The Recognition Rate of proposed method is higher than conventional one. Even though foreign data has been used, the presented method can be applied for data acquired locally. In the next future, this method will be developed to identify appliances that has certain pattern of signal power and can be applied to the smart meter in real time.

We acknowledge the support from Hibah PITTA 2018 funded by DRPM Universitas Indonesia No.2356/UN2.R3.1/ HKP.05.00/2018.

\section{References}

1. P. A. Dahono, konversi ITB, URL: https://konversi.wordpress.com/2011/03/07/ menghemat-energi-dengan-menggunakan-listrik, (2011) [Accessed 1 Jan. 2018].

2. A. Zoha, A. Gluhak, M.A Imram, and S. Rajasegarar, Non-Intrusive Load Monitoring Approaches for Disaggregated Energy Sensing: A Survey, Sensors, Vol.12, Issue 12 (2012).

3. K.E Martinez, K.A Donelly, J.A Laitner, Advanced Metering Initiatives and Residential Feedback Programs: A Meta-Review for Households Electricity-Saving Opportunities, Technical Report of American Council for an Energy-Efficient Economy (2010).

4. Department of Energy \& Climate Change, Energy Consumption in United Kingdom, Technical Report (2010).

5. G. W. Hart, Nonintrusive Appliance Load Monitoring, Proceedings IEEE, Vol. 80, No. 12, (1992).

6. J. G. Roos, I. E. Lane, E. C. Lane,G. P. Hanche, Using Neural Networks For NonIntrusive Monitoring Of Industrial Electrical Loads, Conference Proceedings of 10th Anniversary. IMTC (1994).

7. E.J. Aladesanmi, KA Folly, Overview of non-intrusive load monitoring and identification technique, IFAC-PapersOnLine Vol. 48, Issue 30, p. 415-420 (2015).

8. H.H Chang, K.L Chen, Y.P Tsai, W.J Lee, A New Measurement Method for Power Signatures of Nonintrusive Demand Monitoring and Load Identification, IEEE Transactions on Industry Applications, Vol. 48 (2012).

9. D. Srinivasan, W. S. Ng, A. C. Liew, Neural Network-Based Signature Recognition For Harmonic Source Identification, IEEE Transactions Power Delivery, Vol 21 (2006).

10. Yi Du, Liang Du, Bin Lu, Ronald Harley, Thomas Habetler, A Review of Identification and Monitoring Methods for Electric Loads in Commercial and Residential Buildings, Proceeding of 2010 IEEE Energy Conversion Congress and Exposition (2010).

11. GitHub, areinhardt/tracebase. [online] Available at :https://github.com/areinhardt/ tracebase, (2018) [Accessed 1 Jan. 2018]. 